\title{
Troisième atelier Trattoria consacré au transfert radiatif atmosphérique
}

\author{
Philippe Dubuisson ${ }^{1}$, Adrien Deschamps ${ }^{2}$ \\ 1. Laboratoire d'optique atmosphérique, Université de Lille / CNRS, \\ Villeneuve d'Ascq \\ 2. Cnes, Centre spatial de Toulouse, Toulouse \\ philippe.dubuisson@univ-lille.fr
}

\section{Résumé}

Le Cnes, en collaboration avec le CNRS/Insu et Météo-France, organisait en janvier 2020 à Toulouse la troisième édition de l'atelier Trattoria (Transfert radiatif dans les atmosphères terrestres pour les observations spatiales). Cet atelier est principalement consacré aux codes de transfert radiatif dans l'atmosphère terrestre pour les applications de télédétection spatiale, opérant sur l'ensemble de la gamme des longueurs d'onde de l'ultraviolet aux micro-ondes. Ces codes numériques sont fondamentaux pour la préparation des instruments de télédétection, ainsi que pour le traitement et l'exploitation des données satellitaires. Cet atelier était ouvert à tous les chercheurs, ingénieurs, post-doctorants et doctorants du domaine. Les résultats et recommandations de l'atelier doivent servir de guide au Cnes et aux divers participants et utilisateurs français et européens de codes de transfert radiatif. champs d'application, mais aussi des tables rondes destinées à faire émerger des recommandations pour les développements futurs des différents codes ou des bases de données qui leur sont associées. Une session poster a également été organisée pour permettre aux participants de présenter leurs travaux de recherche ou de développement. d'Ascq en 2015 avec le Laboratoire d'optique atmosphérique de l'université de Lille. Il a été conjointement organisé par le Cnes, le CNRS/Insu et Météo-France, avec pour objectif de réunir les communautés française et européenne francophone intéressées par le transfert radiatif dans l'atmosphère. L'atelier réunissait à la fois des développeurs de modèles ou de codes de transfert radiatif et des utilisateurs de ces outils, pour l'exploitation des données spatiales d'observation de la Terre, ceci sur l'ensemble du spectre électromagnétique de l'ultraviolet aux micro-ondes ${ }^{2}$. Durant les deux jours et demi de l'atelier se sont succédé des présentations orales invitées, visant à dresser le panorama des codes existants, rappeler les récentes évolutions et les verrous existant dans différents

La préparation, l'organisation et l'animation de cet atelier ont été pilotées par un comité de programme, composé de membres du Cnes, de MétéoFrance et de laboratoires du domaine : Raymond Armante (LMD), Philippe Chambon et Jérôme Vidot (CNRM/ Météo-France), Juan Cuesta (Lisa), Philippe Dubuisson (LOA), Adrien Deschamps, Lucas Landier et Pierre Tabary (Cnes) et Céline Cornet (Insu).

Rappelons qu'un code de transfert radiatif est un logiciel permettant de modéliser le transfert et l'émission

1. http://www.meteo.fr/cic/meetings/2020/trattoria/

2. voir La Météorologie, 97, spécial Observation satellitaire, mai 2017.

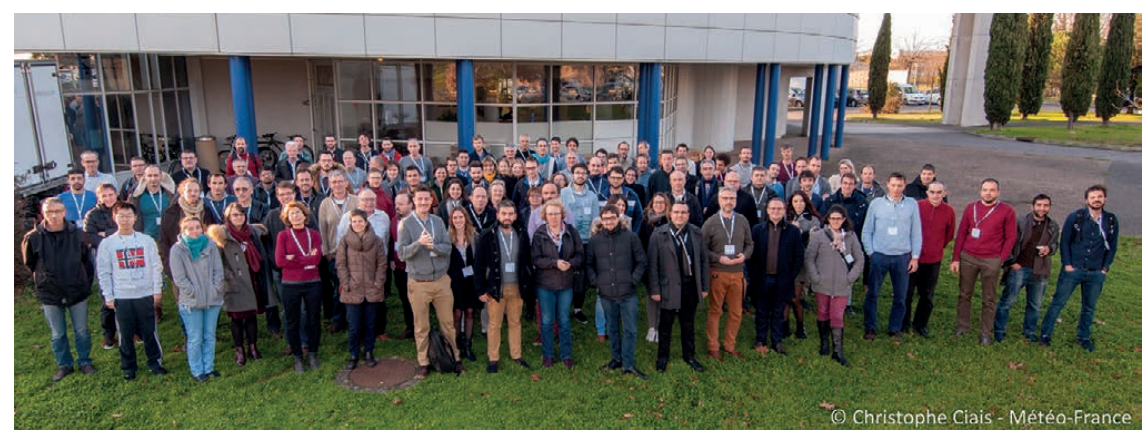

Figure 1. L'atelier Trattoria 2020 a réuni plus d'une centaine de personnes sur la thématique du transfert radiatif. 


\section{Abstract}

\section{Third Trattoria workshop on atmospheric radiative transfer}

The CNES, in collaboration with the CNRS/INSU and Météo-France, organized in January 2020 in Toulouse the third edition of the Trattoria workshop (Transfert radiatif dans les atmosphères terrestres pour les observations spatiales). This workshop is mainly devoted to radiation transfer codes in the Earth's atmosphere for space remote sensing applications, operating over the entire wavelength range from ultraviolet to microwaves. These numerical codes are fundamental for the preparation of remote sensing instruments, as well as for the processing and exploitation of satellite data. This workshop was open to all researchers, engineers, postdoctoral and doctoral students in the field. The results and recommendations of the workshop should serve as a "guide" for CNES and the various French and European participants and users of radiative transfer codes. du rayonnement électromagnétique à travers les atmosphères planétaires. Ce rayonnement, émis par le Soleil ou l'atmosphère et la surface terrestre, peut être réfléchi ou absorbé par les constituants atmosphériques (gaz, nuages et aérosols) ou les surfaces (terrestres ou marines). Le cœur d'un tel code a pour objectif de résoudre mathématiquement l'équation de transfert radiatif à l'aide d'une méthode numérique (voir annexe 1). Il doit prendre en compte l'ensemble des processus radiatifs : absorption, émission, diffusion et polarisation du rayonnement, ainsi que leurs interactions. Couplés à des données décrivant l'état de l'atmosphère, ces codes permettent de calculer en premier lieu la luminance (énergie du rayonnement reçu ou émis dans une direction spécifique par unité de temps, de surface et d'angle solide, exprimée en $\mathrm{Wm}^{-2} \mathrm{sr}^{-1}$ ) en tout point de l'atmosphère. Cette grandeur directionnelle étant ce que mesurent généralement les instruments embarqués sur les satellites, un code de transfert radiatif permet donc de simuler ce que mesure (ou mesurerait) un instrument spatial (simulation d'observables, figure 2). Ces codes permettent aussi de calculer les échanges radiatifs dans l'atmosphère terrestre, en calculant la densité de flux radiatif (énergie radiative traversant un élément de surface par unité de temps, exprimée en $\mathrm{Wm}^{-}$ ${ }^{2}$ ) et donc d'évaluer le pouvoir de réchauffement ou de refroidissement des constituants atmosphériques en tout point de l'atmosphère.

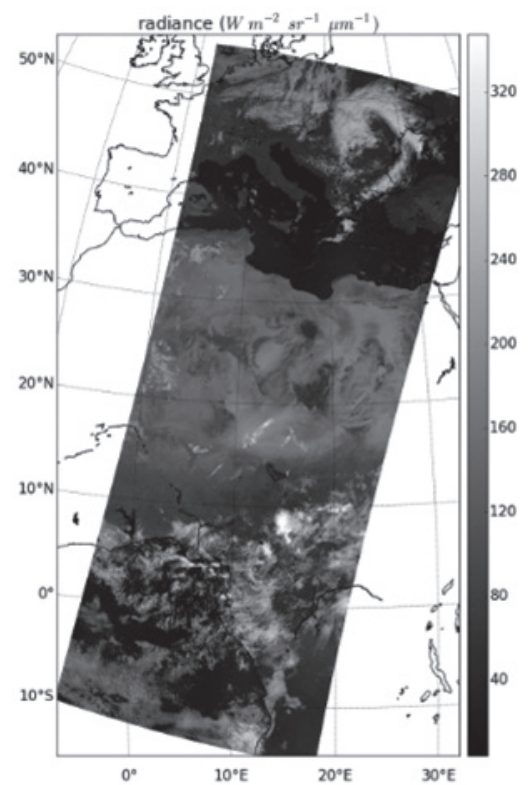

Les codes de transfert radiatif sont souvent utilisés, d'une part, pour aider à la définition des futurs instruments (demandes des agences spatiales) et exploiter les données par la suite, d'autre part comme une brique essentielle des modèles météorologiques ou climatiques. Ils sont souvent également à la base des méthodes d'inversion (estimation optimale, réseaux de neurones, etc.) des mesures des instruments spatiaux. La qualité de ces inversions est le plus souvent fortement liée à la précision de simulation de ces codes (précision de la méthode numérique de résolution, de la simplification de la géométrie choisie, des incertitudes sur les propriétés optiques des aérosols et nuages, de la résolution spectrale, etc.). Il est important que ces codes de transfert radiatif puissent aussi calculer les « jacobiens », c'està-dire les dérivées de l'équation du transfert radiatif (ETR) par rapport aux principaux paramètres dont elle dépend (température, concentration, pression de surface, paramètres aérosols ou nuageux, etc.).

Quelques exemples de codes de transfert radiatif sont donnés dans le tableau 1, avec le lien permettant d'accéder à leur site internet.

Les objectifs de l'atelier étaient les suivants :

- Évaluer les besoins des principaux utilisateurs français et européens (scientifiques, météorologues et climatologues, agences spatiales, sociétés

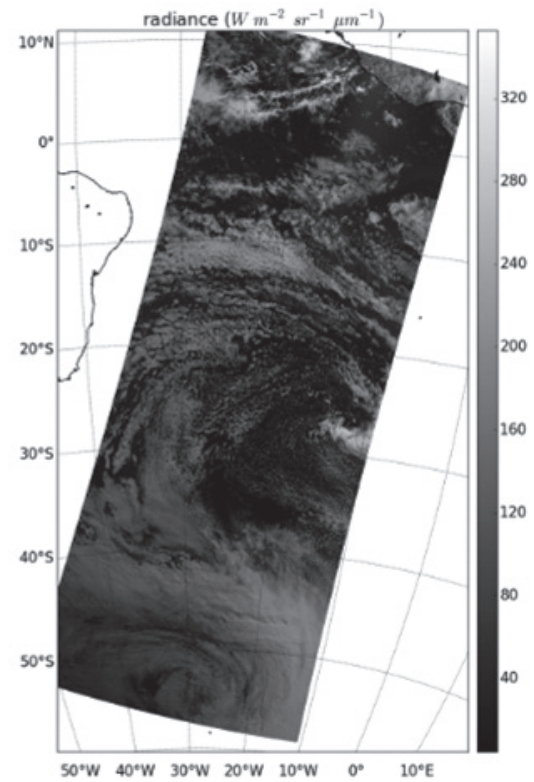

Figure 2. Images synthétiques en luminance spectrale (radiance, en anglais) pour le futur instrument METimage VII-4 (à $752 \mathrm{~nm}$ ) sur la plateforme spatiale EPS-SG d'Eumetsat, créées avec le code de transfert radiatif Artdeco et des données atmosphériques (pression, température, couverture nuageuse, contenu en aérosols, etc.) issues du pôle Aeris/lcare (LOA, Aeris/lcare, Hygeos, projet Eumetsat 4MSDS). 
Tableau 1. Liste non exhaustive de codes de transfert radiatif pour l'atmosphère terrestre. 1D ou 3D : 1 ou 3 dimensions ; IR : Infrarouge, VIS : Visible, IR : Infrarouge, MO : Micro-ondes ; DOM : Discrete Ordinates Method ; SOS : Successive Orders of Scattering, MC : Monte Carlo ; HS : Harmoniques sphériques.

\begin{tabular}{|c|c|c|c|c|c|}
\hline $\begin{array}{l}\text { Nom } \\
\text { du code }\end{array}$ & $\begin{array}{l}\text { Géométrie } \\
\text { de calcul }\end{array}$ & Domaine & $\begin{array}{l}\text { Absorption } \\
\text { spectrale }\end{array}$ & $\begin{array}{l}\text { Résolution } \\
\text { gazeuse }\end{array}$ & $\begin{array}{l}\text { Source internet } \\
\text { de l'ETR }\end{array}$ \\
\hline LBLRTM & $1 \mathrm{D}$ & IR to UV & LBL & DOM & http://rtweb.aer.com/lblrtm.html \\
\hline 4A/OP & $1 \mathrm{D}$ & IR to PIR & LBL & $\mathrm{DOM}$ & http://4aop.aeris-data.fr/ \\
\hline V-Lidort & $1 \mathrm{D}$ & IR to UV & оuі & DOM & http://www.rtslidort.com/mainprod_lidort.html \\
\hline Disort & 10 & IR to UV & oui & DOM & http://en.wikipedia.org/wiki/DISORT \\
\hline RRTM & $1 \mathrm{D}$ & IR to UV & CkDist & DOM & http://rtweb.aer.com/ \\
\hline Streamer & $1 \mathrm{D}$ & IR to VIS & ESFT & DOM & http://stratus.ssec.wisc.edu/streamer/ \\
\hline Modtran & $1 \mathrm{D}$ & IR to UV & CkDist & DOM & http://www.MODTRAN5.com/ \\
\hline $6 S V$ & $1 \mathrm{D}$ & VIS to PIR & Band Model & SOS & http://6s.ltdri.org \\
\hline Matisse & $1 \mathrm{D} / 3 \mathrm{D}$ & IR to VIS & CkDist & & http://matisse.onera.fr/accueil \\
\hline RTTOV & 10 & VIS to MW & Predictors & Predictor & https://nwp-saf.eumetsat.int/site/software/rttov/ \\
\hline Momo & $1 \mathrm{D}$ & UV to IR & CkDist & Matrix operator & Francois.Ravetta@latmos.ipsl.fr / juergen.fischer@fu-berlin.de \\
\hline KCARTA & $1 \mathrm{D}$ & $\mathbb{R}$ & LBL & & https://github.com/strow/kcarta-matlab \\
\hline Kopra & $1 \mathrm{D}$ & $\mathbb{R}$ & LBL & analytical & https://www.imk-asf.kit.edu/english/312.php \\
\hline OSOA-A & $1 \mathrm{D}$ & VIS & & SOS & https://logiciels.cnes.fr/content/osoaa \\
\hline HTRDR & $3 \mathrm{D}$ & IR to VIS & & MC & (https://www.meso-star.com/projects/high-tune/man/man1/htrdr.1.html \\
\hline SHDOM & $3 \mathrm{D}$ & IR to PIR & CkDist & $\mathrm{DOM}+\mathrm{HS}$ & http://nit.colorado.edu/shdom.html \\
\hline 3DMCPOL & $3 \mathrm{D}$ & VIS to IR & CkDist & MC & Celine.Cornet@univ-lille.fr \\
\hline
\end{tabular}

Librairies dédiées au transfert radiatif avec un ensemble de données, codes et documents

\begin{tabular}{lrr} 
LIBRADTRAN & LIBrary for RADiative TRANsfer & http://www.libradtran.org/doku.php?id=start \\
\hline Artdeco & Atmospheric Radiative Transfer Database for Earth Climate Observation & http://loa-git.univ-lille1.fr/wikiloa_public/doku.php?id=artdeco:start \\
\hline CRTM & Community Radiative Transfer Model & https://www.jcsda.org/jcsda-project-community-radiative-transfer-model \\
\hline
\end{tabular}

d'algorithmie) en code de transfert radiatif dans les cinq ou dix prochaines années. Le champ d'application de cet atelier était limité à l'atmosphère terrestre.

- Dresser un panorama des codes actuellement utilisés en les classant selon leurs principales caractéristiques, présenter leurs principales évolutions depuis l'atelier précédent et identifier leurs utilisations dans différents champs d'application.

- Faire se rencontrer les différentes communautés utilisatrices et inciter à une plus grande convergence des codes utilisés en France, au moins par domaine spectral et pour les modèles de grande précision.

- Mieux faire connaître les logiciels développés et les bases de données associées, en particulier ceux réalisés avec le concours du Cnes pour ses propres missions scientifiques.

\section{Déroulement de l'atelier}

L'atelier se composait d'exposés de synthèse, de posters et de tables rondes, afin de dresser le panorama des différentes méthodes ou modèles existant dans les codes français et internationaux, avec si possible quelques informations sur l'organisation et les moyens mis en œuvre pour assurer la maintenance et le développement des codes. Les exposés de synthèse (sur invitation) étaient destinés à présenter l'état de l'art de chaque domaine, rappeler les enjeux scientifiques, les principaux concepts physiques, les problèmes à résoudre et les évolutions attendues pour les prochaines années. Les tables rondes permettaient ensuite de débattre autour de sujets clés et de faire émerger des conclusions et recommandations. Des posters venaient compléter les exposés, pour proposer un approfondissement sur ce qui est à l'étude dans les laboratoires ou réalisé par les différents acteurs (laboratoires, sociétés privées, agences spatiales, etc.). Enfin, des tutoriels ont également été organisés pour présenter quelques codes de transfert radiatif (OSOAA, 4A/OP, Artdeco et RTTOV) utilisés dans ces communautés. Les présentations et tables rondes sont listées dans le tableau 2 , avec un résumé proposé en annexe pour celles qui sont indiquées en gras.

\section{Bilan et \\ recommandations}

L'atelier a réuni une audience plus importante que celle des précédentes éditions (130 personnes contre une centaine en 2015 et moins de 70 en 2008), soulignant ainsi l'intérêt croissant de la communauté scientifique pour le transfert radiatif, mais également celui d'un certain nombre d'industriels souhaitant développer des applications innovantes dans l'utilisation de données spatiales.

Les présentations ont permis de dresser un état de l'art des principaux travaux menés dans ce domaine ces cinq dernières années. On peut noter des efforts soutenus pour améliorer le temps de calcul des codes (avec différentes stratégies : portage sur GPU, parallélisation massive, simplification des modèles de diffusion...), mais également pour prendre en compte les effets tridimensionnels ou, pour les données avec la plus haute résolution spectrale, des effets fins de spectroscopie. Les applications spatiales étaient au cœur des discussions et les différentes contributions ont 
montré comment, pour de nombreuses missions, la modélisation du transfert radiatif dans l'atmosphère pouvait constituer un verrou difficile à lever.

Bien évidemment, les axes de recherche visant à améliorer les codes existants dépendent souvent des usages qu'on souhaite faire de la donnée spatiale, car ceux-ci peuvent être très nombreux. On a vu en effet, lors de cet atelier, que le transfert radiatif était un élément clef à la fois pour les capteurs classiquement dédiés à l'étude de l'atmosphère (les spectromètres infrarouge, les lidars), mais également pour des radiomètres imageurs ou des spectroradiomètres dédiés à l'étude de la végétation, des sols ou du littoral. C'est pourquoi, pour établir les recommandations qui serviront à guider la communauté française pour les cinq prochaines années, dans ses efforts autour du transfert radiatif atmosphérique, des tables rondes thématiques ont été organisées.

Cependant, certains constats dressés lors de cet atelier sont transverses et ont une portée générale. En particulier, il est apparu que la communauté française a une expertise dans le transfert radiatif qui est reconnue à l'échelle européenne et internationale. Cependant, il a aussi été souligné que ces compétences devaient être maintenues de façon pérenne et qu'un effort dans ce sens était nécessaire de la part des différentes tutelles des laboratoires de recherche. À cet effet, et pour davantage coordonner les travaux de la communauté, un groupement de recherche « transfert radiatif » pourrait être créé. Les recommandations émises par les différents groupes de travail seront
Tableau 2. Liste des présentations et tables rondes.

\begin{tabular}{|c|c|}
\hline Titre & Intervenants (affiliation) \\
\hline \multicolumn{2}{|c|}{ Présentations orales } \\
\hline $\begin{array}{l}\text { Présentation introductive } \\
\text { et programmatique }\end{array}$ & A. Deschamps (Cnes), P. Tabary (Cnes) et E. Defer (LA) \\
\hline $\begin{array}{l}\text { Panorama des codes de transfert radiatif } \\
\text { et état des derniers développements }\end{array}$ & R. Armante (LMD) et P. Dubuisson (LOA) \\
\hline $\begin{array}{l}\text { De l'intérêt d'une bonne spectroscopie } \\
\text { pour connaître l'atmosphère }\end{array}$ & J.-M. Hartmann (LMD) et E. Turner (MetOffice) \\
\hline Diffusion et particules & H. Herbin (LOA) et P. Chambon (CNRM) \\
\hline $\begin{array}{l}\text { Approches directe/inverse pour la restitution } \\
\text { couplée de la composition atmosphérique } \\
\text { et les propriétés de la surface }\end{array}$ & J. Cuesta (Lisa) et D. Carrer (CNRM) \\
\hline
\end{tabular}

\begin{tabular}{ll}
\hline $\begin{array}{l}\text { Application à la météorologie opérationnelle } \\
\text { et à la modélisation du climat }\end{array}$ & Q. Libois et J. Vidot (CNRM) \\
\hline $\begin{array}{l}\text { Modélisation tridimensionnelle du transfert } \\
\text { radiatif pour des scènes terrestres naturelles }\end{array}$ & P. Chervet (Onera) et F. Szczap (LaMP) \\
\hline
\end{tabular}

radiatif pour des scènes terrestres naturelles

\begin{tabular}{ll}
\hline Utilisation du transfert radiatif & C. Pierangelo (Cnes) et B. Fougnie (Eumetsat) \\
par les agences spatiales &
\end{tabular}

\begin{tabular}{lc}
\multicolumn{2}{c}{ Tables rondes } \\
Particules : aérosols et nuages & J. Riedi (LOA) et P. Formenti (Lisa) \\
\hline Lien entre modèles et transfert radiatif & V. Guidard (CNRM) et L. Landier (Cnes) \\
\hline Instruments actifs : lidar et radar & V. Noël (LA) et P. Chambon (CNRM) \\
\hline Codes de transfert radiatif rapides & C. Cornet (LOA) et J. Vidot (CNRM) \\
\hline Spectroscopie & C. Crevoisier (LMD) et A. Deschamps (Cnes) \\
\hline Corrections atmosphériques & M. Chami (Latmos) et O. Hagolle (Cesbio) \\
\hline Cesbio, Centre d'études spatiales de la biosphère & Lisa, Laboratoire interuniversitaire des systèmes \\
$\begin{array}{l}\text { Eumetsat, Organisation européenne pour l'exploitation } \\
\text { des satellites météorologiques }\end{array}$ & atmosphériques \\
LA, Laboratoire d'aérologie & LMD, Laboratoire de météorologie dynamique \\
LaMP, Laboratoire de météorologie physique & LOA, Laboratoire d'optique atmosphérique \\
Latmos, Laboratoire atmosphères, milieux, observations & MetOffice, Meteorological Office \\
Onera, Office national d'études et de recherches aérospatiales
\end{tabular}

spatiales

endossées conjointement par le Cnes et l'Insu et transmises à un certain nombre de comités scientifiques ou de pilotage.

Enfin, décision a été prise de renouveler l'expérience Trattoria et d'organiser une quatrième édition à l'horizon 2024.

\section{Pour en savoir plus :}

Présentations effectuées lors de Trattoria 2019 : http://www.meteo.fr/ cic/meetings/2020/trattoria/ Présentations de Trattoria 2015 : http:// www.meteo.fr/cic/meetings/2020/ trattoria/trattoria2015.html ou http:// Trattoria-2015/ www-loa.univ-lille1.fr/workshops/ 


\section{Annexes}

\section{Panorama des codes de transfert radiatif et état des derniers développements}

Le transfert radiatif (Herbin et Dubuisson, 2016) permet de décrire l'interaction du rayonnement électromagnétique avec la matière et d'analyser la propagation de ce rayonnement à travers les milieux gazeux, solides ou liquides. Au cours de sa propagation, le rayonnement subit des variations que l'on peut traduire mathématiquement par une équation intégro-différentielle, c'est-à-dire contenant des dérivées et intégrales par rapport au temps, aux variables de position et à la direction de propagation : c'est l'équation de transfert du rayonnement (ETR). Dans un milieu atmosphérique, l'ETR a pour objectif de décrire la propagation du rayonnement en prenant en compte les phénomènes d'absorption, de diffusion et d'émission thermique (voir annexes 2 et 3$)$.

Un code de transfert radiatif atmosphérique est un logiciel calculant les grandeurs radiatives (Dubuisson, 2017) en tout point d'une atmosphère planétaire à partir de la résolution mathématique de l'ETR, prenant aussi en compte la perturbation du rayonnement provoquée par l'instrument

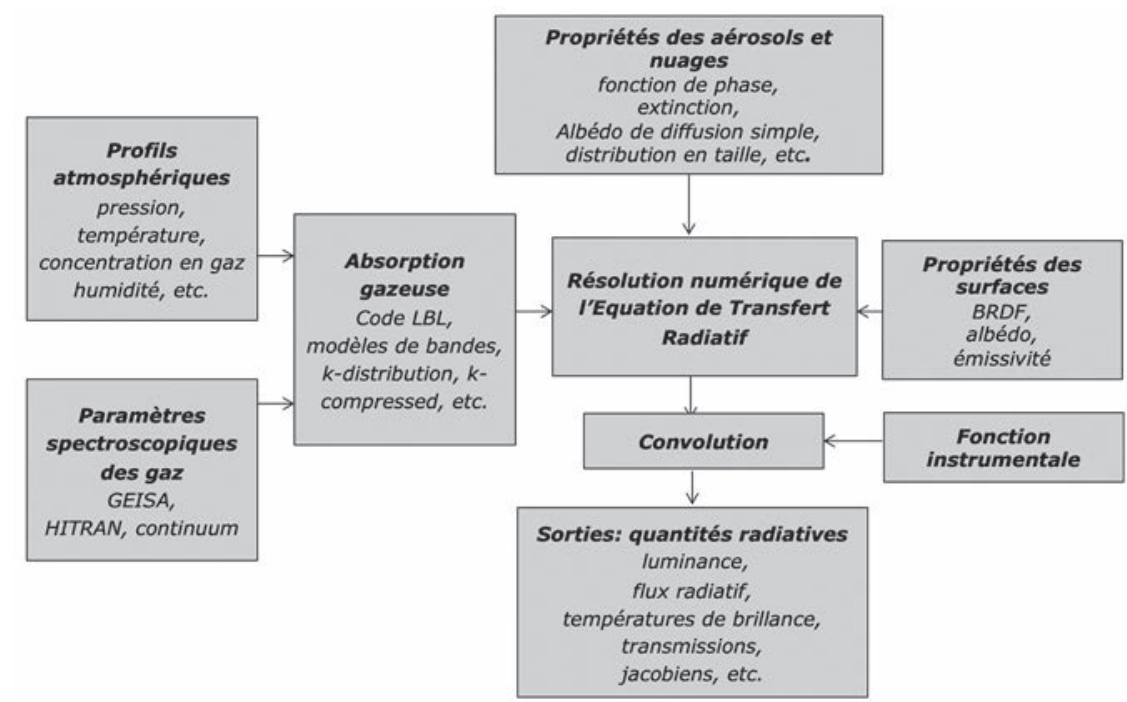

Figure 1.1. Diagramme général pour la résolution de l'équation de transfert radiatif.

(convolution par la fonction instrumentale) que l'utilisateur souhaite simuler. Ces codes sont extrêmement importants en télédétection pour des études préparatoires, la définition des instruments, des études de bilan d'erreur, l'étalonnage, la validation des instruments et la mise au point des méthodes inverses.
Le grand nombre de missions spatiales futures et existantes, ainsi que les travaux de validation et d'étalonnage associés, nécessitent des codes de plus en plus performants. La nature des mesures en cours ou à venir requièrent aussi des codes de plus en plus performants, capables de prendre en compte les nombreuses spécificités

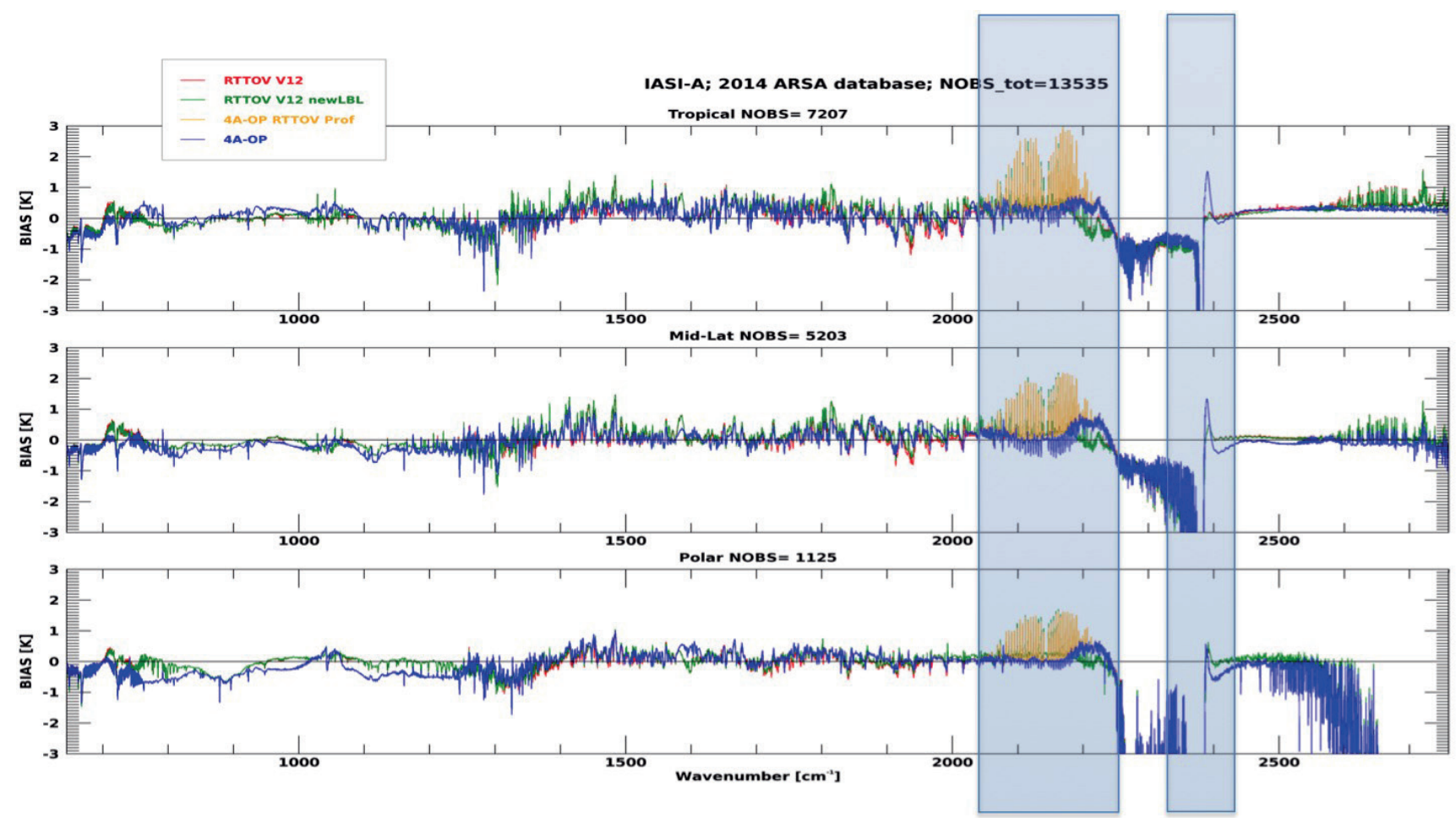

Figure 1.2. Intercomparaison entre les codes 4AOP et RTTOV et les observations de l'instrument spatial à haute résolution spectrale lasi. Les biais (écarts entre les observations et les simulations des codes) sont représentés en température de brillance (K) en fonction du nombre d'onde $\left(\mathrm{cm}^{-1}\right)$. 
de ces capteurs : gamme et résolution spectrales (UV, visible, infrarouge et micro-ondes), calculs de jacobiens, nature des mesures (radar, lidar, radiomètre, spectromètre), polarisation, géométrie d'observation. Enfin, avec des besoins en précision (radiométriques et/ou spectrales) grandissant, les codes doivent prendre en compte des phénomènes physiques de plus en plus fins (paramètres spectroscopiques par exemple, annexe 2). Un code universel étant difficile à concevoir, il est souvent nécessaire d'utiliser simultanément plusieurs codes pour répondre à ces besoins. Cependant, un compromis sera souvent nécessaire entre la précision et la rapidité des calculs, selon les objectifs à atteindre.

La résolution précise de l'ETR, en prenant en compte l'ensemble des processus radiatifs (émission, absorption, diffusion) dans toutes les directions, est un problème compliqué, car la répartition spatiale des certains composants atmosphériques est très hétérogène et les conditions aux limites complexes (en particulier les surfaces). En outre, le problème fait intervenir cinq dimensions : trois variables d'espace et deux variables angulaires. Des solutions analytiques exactes n'existant pas - sauf pour des cas très idéalisés-, des méthodes numériques sont nécessaires. On distingue deux grandes familles de codes :

- Les méthodes statistiques tridimensionnelles, de type Monte Carlo, calculent les trajectoires des rayons lumineux à l'aide de procédures aléatoires (voir annexe 5). Cette approche permet d'explorer toutes les géométries et tient compte des hétérogénéités spatiales, ce que les méthodes explicites ne permettent pas. La précision

1. Voir par exemple pour les codes 3D le site internet de l'International Intercomparison of $3 \mathrm{D}$ radiative transfer code (I3RC) : http://i3rc. gsfc.nasa.gov dépend du nombre de trajectoires simulées et, par conséquent, elle est directement liée au temps de calcul. La puissance des ordinateurs actuels et le développement de nouvelles techniques numériques, telles que le calcul parallèle ou les cartes graphiques, rendent désormais plus confortable l'utilisation de ces codes.

- Les méthodes explicites résolvent l'ETR sur l'ensemble du champ de rayonnement, discrétisé pour le calcul en un certain nombre de directions, et pour une atmosphère découpée en couches planes, parallèles et homogènes. Cette représentation simplifiée de l'atmosphère, souvent notée 1D, permet de résoudre l'ETR rapidement à l'aide de méthodes mathématiques telles que les ordonnées discrètes (Discrete Ordinates Method ou DOM), les ordres successifs de diffusion (Successive Orders of Scattering ou SOS), les harmoniques sphériques, etc. Elles sont le plus souvent utilisées pour des applications nécessitant un grand nombre de simulations, telles que la modélisation climatique ou le traitement opérationnel des données satellitaires.

Tant pour la précision que pour la rapidité de calcul, un autre point délicat est le traitement de l'absorption gazeuse. La méthode la plus précise, mais aussi la plus coûteuse en temps de calcul, consiste à utiliser une approche dite « raie-par-raie » (line-by-line ou LBL), qui prend en compte chaque raie d'absorption de chaque gaz avec une haute résolution spectrale (voir annexe 2). Pour diminuer le temps de calcul, des méthodes approchées peuvent être utilisées, souvent à partir de paramétrisations, soit pour éviter de faire la sommation de toutes les raies d'absorption ( $k$-distribution ou corrélation), atlas de données (Look-Up Table, etc.), soit pour éviter de faire la convolution par la fonction d'instrument (régression, etc.).
La figure 1.1 donne une représentation schématique du fonctionnement de ces méthodes de résolution de l'ETR et des données d'entrée dont il faut généralement disposer.

Un point important, qu'il est nécessaire de continuer à soutenir dans le futur concerne la validation de ces codes, tant pour regarder leur précision que pour définir les processus qui nécessitent d'être améliorés. Des exercices de validation et/ou d'intercomparaison de codes sont régulièrement effectués ${ }^{1}$. À titre d'exemple, la figure 1.2 présente une récente comparaison des simulations des codes RTTOV et 4AOP avec les mesures du spectromètre Iasi. Cette intercomparaison a permis de mettre en évidence que RTTOV avait un défaut d'absorption du monoxyde de carbone (CO) vers $2150 \mathrm{~cm}^{-1}$, alors que 4A/OP avait un problème d'absorption du dioxyde de carbone $\left(\mathrm{CO}_{2}\right)$ vers $2350 \mathrm{~cm}^{-1}$. Ces points ont pu ainsi être identifiés, puis corrigés dans les versions suivantes.

Longtemps délaissés, car considérés comme suffisamment précis, les besoins des nombreuses missions spatiales actuelles et futures nous rappellent que ces codes doivent continuer à être sans cesse actualisés et améliorés. Le besoin de soutien humain sur ces codes est primordial afin d'en assurer la maintenance, le suivi et l'amélioration, mais aussi nécessite une expertise qui, nous sommes nombreux à l'avoir constaté, est en train de disparaître tant au niveau national qu'international.

Raymond Armante
Philippe Dubuisson
${ }^{2}$
1. Laboratoire de météorologie dynamique,
Palaiseau
2. Laboratoire d'optique atmosphérique,
Université de Lille / CNRS, Villeneuve d'Ascq

Raymond Armante ${ }^{1}$ Philippe Dubuisson ${ }^{2}$ Palaiseau Université de Lille / CNRS, Villeneuve d'Ascq 


\section{De l'intérêt d'une bonne spectroscopie pour connaître l'atmosphère}

La spectroscopie, par sa composante qui vise à modéliser comment les gaz interagissent avec la lumière, est un outil précieux pour l'étude du système Terre. Elle permet, via des calculs de transfert radiatif, de simuler l'absorption et l'émission atmosphérique et, par l'ajustement des spectres calculés sur ceux mesurés par divers instruments (au sol, sous ballons, sur des satellites), de déduire certaines caractéristiques de l'atmosphère. Il est dès lors évident que la précision des résultats fournis par de telles procédures d'« inversion » est conditionnée par la fiabilité des informations spectroscopiques utilisées, et qu'il faut calculer au mieux l'amplitude et la forme des spectres. Il s'agit là d'un défi, car les collisions entre molécules modifient le profil d'absorption en faisant intervenir des processus physiques complexes et variés qui dépendent de la région spectrale étudiée. De nombreux travaux expérimentaux et théoriques de laboratoire ont donc étudié l'influence de la pression sur les raies isolées, sur des «paquets » de raies proches, ainsi que sur les continuums à variation spectrale lente dus aux ailes lointaines des raies et à l'absorption induite par collision (Hartmann et al., 2008).

\section{Les gaz à effet de serre}

La bande $\mathrm{A}$ de l'oxygène moléculaire (vers $0,76 \mu \mathrm{m}$, figure 2.1) est un exemple de large région du spectre atmosphérique où plusieurs processus doivent être pris en compte avec précision puisqu'elle sera utilisée pour déterminer la pression de surface à partir des spectres enregistrés par le spectromètre satellitaire MicroCarb ${ }^{1}$ du Cnes qui doit cartographier le $\mathrm{CO}_{2}$ atmosphérique à partir de ses signatures vers 1,6 et $2 \mu \mathrm{m}$. On voit en effet sur la figure 2.1 que ne prendre en compte que les contributions des raies individuelles conduit à des erreurs importantes, et que les résultats sont bien meilleurs lorsque le modèle inclut aussi l'absorption induite par collision et les effets (de line-mixing) associés aux échanges collisionnels de population entre niveaux de rotation.

Un autre exemple, qui concerne une région beaucoup plus étroite vers $1,6 \mu \mathrm{m}$, est celui d'un multiplet constitué de plusieurs transitions du méthane qui a été retenu pour la future mission
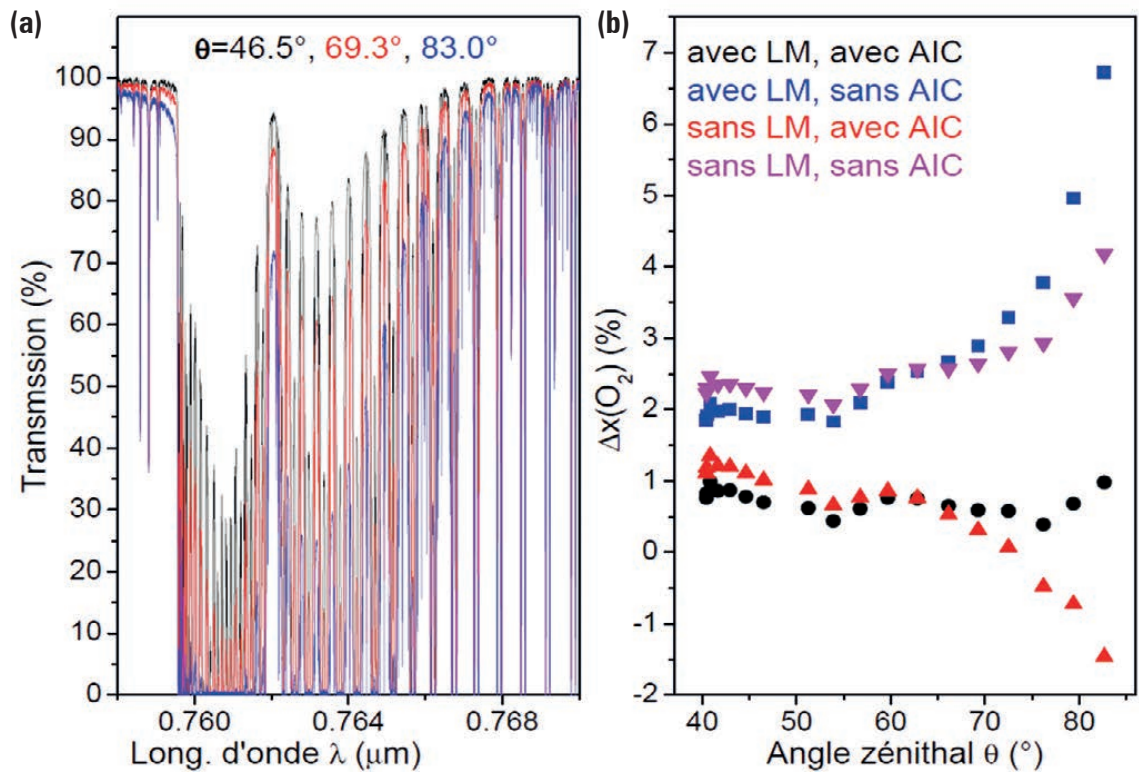

Figure 2.1. (a) Transmissions atmosphériques dans la région de la bande $\mathrm{A}$ de $\mathrm{O}_{2}$ mesurées du sol pour différents angles zénithaux $\theta$. (b) Erreur sur la fraction molaire de $0_{2}$ obtenue par inversion de spectres tels que ceux de la figure (a) en utilisant des modèles prenant ou non en compte le line-mixing (LM) et l'absorption induite par collision (AIC). D'après Tran et Hartmann (2008).

satellitaire franco-allemande Merlin (Erhet et al., 2017) pour la mesure du méthane atmosphérique. Les effets précités de line-mixing doivent ici encore être pris en compte, mais aussi ceux, plus complexes, associés aux changements de la vitesse des molécules dus aux collisions ainsi qu'à l'influence de cette vitesse sur le profil des raies (Delahaye et al., 2016).

Pour que les projets MicroCarb et Merlin fournissent des informations atmosphériques (respectivement des quantités de $\mathrm{CO}_{2}$ et $\mathrm{CH}_{4}$ ) pertinentes, l'erreur dans la simulation des spectres enregistrés doit être meilleure que $0,3 \%$. Ce chiffre démontre l'importance d'une " bonne spectroscopie » et à quel point cette discipline de recherche est aujourd'hui sollicitée, puisqu'elle doit atteindre des précisions dix fois meilleures que par le passé.

\section{La vapeur d'eau et son continuum aux ondes submillimétriques}

La caractérisation de l'absorption par la vapeur d'eau dans l'atmosphère est d'une importance cruciale pour la prévision à long terme des changements climatiques et celle, à court terme, des conditions météorologiques. La télédétection à partir de satellites en orbite autour de la Terre contribue ainsi de manière significative à la précision des modèles. Les raies d'absorption dans la partie micro-ondes (fréquences $<200$ $\mathrm{GHz}$ ) et infrarouge (longueurs d'onde $<15 \mu \mathrm{m})$ du spectre sont utilisées depuis longtemps pour retrouver la répartition verticale de la vapeur d'eau. Cependant, les premières mesures ont détecté, en plus des transitions étroites bien connues, une absorption " anormale ». Cette dernière, qui sera ultérieurement appelée continuum, varie lentement avec la longueur d'onde et son origine n'a pas encore été complètement expliquée. La région submillimétrique au-dessus de $200 \mathrm{GHz}$ a reçu peu d'attention en science atmosphérique en raison de la rareté des mesures, mais cela changera avec le lancement (prévu en 2023) du satellite MetOp$S G$ B. Il transportera l'instrument Ice Cloud Imager (ICI), qui sondera l'atmosphère dans 13 canaux sensibles à la vapeur d'eau entre 183 et $664 \mathrm{GHz}$. Les canaux proches de $243 \mathrm{GHz}$ sont particulièrement influencés par le continuum, et l'interprétation précise des futures mesures met en question la spectroscopie dans cette partie relativement inexplorée du spectre. Deux théories ont été proposées pour expliquer le continuum, qui font encore débat : les contributions des dimères, dont des transitions ont été détectées récemment aux basses fréquences, et

1. https://fr.wikipedia.org/wiki/MicroCarb 
celles des ailes des raies d'absorption du monomère qui seraient inadéquatement décrites par les profils spectraux usuels. Des travaux ultérieurs ayant montré que les dimères ne permettent pas d'expliquer tout le continuum, une correction a été apportée aux ailes des raies du monomère pour tenir compte de l'absorption manquante (Serov et al., 2017). Alternativement, un groupe de la société américaine AER développe, depuis plus de 30 ans, des paramétrisations du continuum entièrement fondées sur les ailes des raies du monomère et aujourd'hui dénommée MT-CKD (Mlawer et al., 2012), qui est la référence actuelle pour décrire le continuum pour toutes les parties du spectre. Ses évolutions les plus récentes font suite à des mesures au-dessus de $650 \mathrm{GHz}$ obtenues pendant la campagne RHUBC-II au Chili et autour de la raie à $183,31 \mathrm{GHz}$ (Mlawer et al., 2019), mais la région submillimétrique entre ces deux fréquences n'a jamais été directement étudiée.

Les codes de transfert radiatif rapides, tels que RTTOV (Saunders et al., 2018), permettent d'atteindre des vitesses de calcul plus élevées en utilisant des paramètres satellites précalculés par des codes raie par raie. Amsutran en est un exemple (Turner et al., 2019) qui est utilisé pour tous les instruments hyperfréquences simulés par RTTOV. $\mathrm{Sa}$ spectroscopie, qui repose sur le modèle MPM89 (Liebe, 1989), utilise un continuum de la vapeur d'eau obtenu à partir de mesures de laboratoire à $137 \mathrm{GHz}$, qui pourraient être améliorées pour le sous-millimétrique. Une comparaison des luminances en haut de l'atmosphère obtenues avec différents modèles de continuum indique une incertitude associée à ce dernier d'environ $1 \mathrm{~K}$ (en moyenne et en unités de température de brillance) dans la fenêtre 200-300 GHz (Turner et al., 2019). Les capacités de simulation submillimétriques seraient sans aucun doute améliorées par davantage de mesures pour réduire ces incertitudes. Une voie à suivre pourrait également être de glaner des connaissances auprès d'autres communautés plus établies dans la région des térahertz, comme celles de l'astronomie ou de l'optique quantique. De même, les enseignements tirés lorsque l'ICI sera en orbite pourraient être partagés et ainsi bénéficier à divers domaines scientifiques, afin de progresser vers une description théorique complète $\mathrm{du}$ continuum de la vapeur d'eau.

\section{Jean-Michel Hartmann ${ }^{1}$} Emma Turner ${ }^{2}$

1. Laboratoire de météorologie dynamique, Palaiseau

2. Met Office, Exeter, Royaume-Uni

Delahaye T. et al., 2016. Precise methane absorption measurements in the $1.64 \mu \mathrm{m}$ spectral region for the MERLIN mission. J. Geophys. Res. Atmos. $121,7360-7370$.

Erhet G. et al., 2017. MERLIN: A French-German space lidar mission dedicated to atmospheric methane. Remote Sens., 9, 1052.

Hartmann J.-M., Boulet C., Robert D., 2008. Collisional effects on molecular spectra: Laboratory experiments and models, consequences for applications. Elsevier.

Liebe H.J., 1989. MPM-An atmospheric millimeter-wave propagation model. Int. J. Infrared Millim. Waves, 10, 631-650.

Mlawer E. et al., 2012. Development and recent evaluation of the MT_CKD model of continuum absorption. Phil. Trans. R. Soc., 370A, 2520-2556.

Mlawer E. et al., 2019. Analysis of water vapor absorption in the far-infrared and submillimeter regions using surface radiometric measurements from extremely dry locations. J. Geophys. Res: Atmos., 124, 8134-8160.

Saunders E.R. et al., 2018. An update on the RTTOV fast radiative transfer model (currently at version 12). Geophys. Model Dev., $11,2717-2737$.

Serov E. et al., 2017. On the origin of the water vapor continuum absorption within rotational and fundamental vibrational bands. J. Quant. Spectrosc. Radiat. Transf., 193, 1-12.

Tran H., Hartmann J.-M., 2008. An improved $\mathrm{O}_{2}$ A band absorption model and its consequences for retrievals of photon paths and surface pressures, $J$. Geophys. Res., 113, D18104. doi: 10.1029/2008JD010011

Turner E. et al., 2019. AMSUTRAN: A microwave transmittance code for satellite remote sensing. J. Quant. Spectrosc. Radiat. Transf., 227, 117-129.

Turner E. et al., 2019. Sub-millimetre spectroscopy for AMSUTRAN. Part One: The Theoretical Basis. NWP SAF report, NWPSAF-MO-TR-038. Disponible à https://www.nwpsaf.eu/publications/tech_reports/amsutran_1Thz_NWPSAF_report.pdf 


\section{Diffusion et particules}

Prendre en compte l'effet de particules dans la simulation d'observations spatiales est une tâche complexe. La stratégie mise en œuvre pour modéliser ces effets repose souvent sur une montée progressive d'échelles: de l'échelle de la particule individuelle vers l'échelle de la mesure spatiale. L'échelle des observations spatiales est typiquement kilométrique, tandis que l'échelle des particules est de quelques nanomètres pour les aérosols organiques secondaires, micrométrique pour les aérosols terrigènes, les gouttelettes et les cristaux de glace ou millimétrique pour les gouttes de pluie par exemple.

Ainsi, on va d'abord modéliser les interactions entre le rayonnement électromagnétique (d'origine solaire ou tellurique en télédétection passive ou lidar, radar en télédétection active) et une particule. À ce stade, de nombreux paramètres influent sur la nature des interactions radiatives, notamment la forme ou morphologie de la particule, sa taille, sa densité ou encore sa composition chimique ou minéralogique.

Puis, dans un second temps, on va intégrer ces propriétés radiatives, en prenant en compte la répartition (en nombre et en taille) de ces particules dans un volume compatible avec l'échelle mesurée par le satellite. Cette démarche en deux temps repose sur l'idée que seul l'effet de groupe des particules est observable depuis l'espace.

Que ce soit pour la génération de produits de niveaux 2 ou l'assimilation des données dans les modèles (chimie-transport, météorologique, climatique), de nombreux efforts ont porté ces dernières années sur le développement de méthodes numériques (voir annexe 1) de résolution de l'équation de transfert radiatif (ordres successifs de diffusion, ordonnées discrètes, adding-doubling, méthode Monte-Carlo, etc.). L'utilisation d'une méthode ou d'une autre relève souvent d'un compromis entre précision désirée et temps de calcul. De plus, si ces méthodes sont parfaitement maîtrisées, elles nécessitent de nombreuses données ancillaires dont la méconnaissance constitue la principale source d'incertitude.

En effet, la détection, la caractérisation physico-chimique et la quantification des particules en suspension nécessitent la connaissance précise de leurs propriétés optiques. À titre de comparaison, cette caractérisation pour la télédétection des espèces gazeuses correspond aux mesures spectroscopiques de références de la position et de l'intensité absolue des raies d'absorption. En particulier, l'indice complexe de réfraction (ICR) est un paramètre essentiel des mesures à distance, car il permet de faire le lien entre la nature chimique, les paramètres microphysiques et les propriétés optiques des particules. Or, ces ICR ne sont connus, au mieux, que pour quelques espèces et quelques longueurs d'onde, et sont généralement déterminés à partir de matériaux massifs ou en lames minces. Ce paramètre est une des principales sources d'incertitude pour la restitution des paramètres des aérosols par télédétection. En effet, il a d'une part été montré que les ICR sont inappropriés pour l'étude de l'interaction rayonnement-matière dans un milieu diffusant constitué de particules en suspension (McPeath et al., 2002). Il a d'autre part été mis en évidence que l'extrapolation de leurs valeurs à quelques longueurs d'onde sur tout le spectre est difficile (Herbin et al., 2017). De plus, la dépendance de l'ICR à d'autres paramètres tels que la température, l'humidité relative ou encore le vieillissement des aérosols sont des propriétés encore mal connues. Des travaux théoriques et expérimentaux récents devraient toutefois permettre de répondre à cette problématique ( $\mathrm{Di}$ Biagio et al., 2014 ; Reed et al., 2018 ; Deguine et al., 2020). Ils permettront d'alimenter des bases de données exploitées par les codes de transfert radiatif avec des propriétés radiatives précises.

La forme des différents types de particules, aérosols, cristaux de neige ou de glace, est également extrêmement variée dans la nature. Il est possible de documenter ces formes pour chaque type de particule, à partir d'échantillons recueillis pendant des campagnes de mesures, par exemple. La petite taille de certains aérosols nécessite d'utiliser un microscope à balayage électronique, tandis que certains flocons de neige peuvent être scannés par des systèmes de caméras avancées. Les distributions en taille de particules peuvent également être échantillonnées avec des instruments de mesure de type APS (Aerosol Particle Sizer) ou SMPS (Scanning Mobility Particle Sizer). Toutefois, celles-ci sont caractérisées par un lien important avec la forme des particules, car les distributions sont en général paramétrisées en fonction d'un facteur de forme et de densité. De plus, le facteur de forme se révèle être souvent très réducteur de la forme réelle d'une particule, en particulier dans le cas de particules très asymétriques. Ainsi, bien que le traitement théorique et numérique de l'effet de la forme et de l'orientation des particules sur la diffusion soit efficacement réalisé par le biais de code ad hoc (théorie de Mie pour les sphères, T-matrice pour les sphéroïdes, Discrete Dipole Approximation ou méthode des éléments finis pour les particules plus complexes), le problème de représentativité par rapport à des mesures d'échantillons réels se pose toujours. Par exemple, la figure 3.1 illustre la forte sensibilité des simulations de températures de brillance à $183,31 \mathrm{GHz}$ à la forme de particule choisie pour représenter la neige dans le transfert radiatif.

Selon le type de particules, aérosols ou bien constituées d'eau condensée, la documentation de leurs propriétés radiatives en fonction du domaine de longueur d'onde se révèle être à un stade assez différent. En effet, contrairement aux aérosols, le nombre de bases de données dédiées aux cristaux de neige et de glace a littéralement explosé ces vingt dernières années (Kneifel et al., (a)

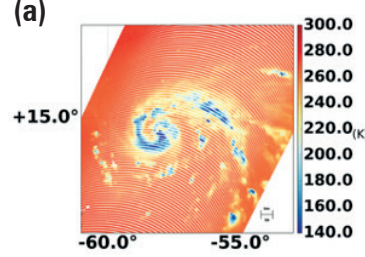

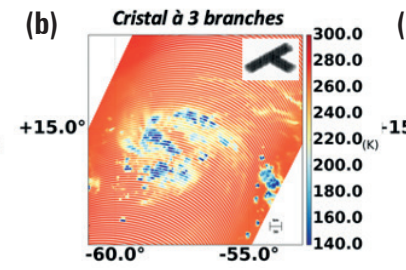

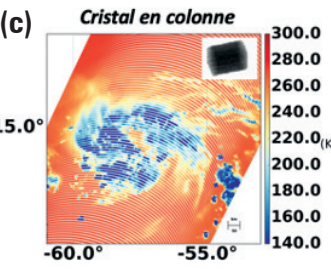

Figure 3.1. (a) Températures de brillance observées, issues du radiomètre micro-ondes GMl à bord du satellite américano-japonais GPM-Core, pour le canal 183,31 \pm 7 GHz. Le cyclone observé est le cyclone Maria dans le bassin de l'Atlantique Nord, le 18 septembre 2017. (b) et (c) Températures de brillance simulées à partir de prévisions du modèle Arome-Antilles de Météo-France. Les simulations ont été réalisées avec le code de transfert radiatif RTOV-SCATT Version 12, pour deux formes de cristaux différentes afin de représenter les propriétés radiatives de la neige. 
2018). À la fin des années 1990 , seuls des cristaux de formes simples étaient considérés dans le transfert radiatif. Depuis, ce sont des formes plus complexes comme celles des agrégats de neige et des particules partiellement fondues dont les effets radiatifs sont modélisés (par exemple, Eriksson et al., 2018). Certaines bases de données prennent même en compte leurs effets complexes sur les ondes électromagnétiques polarisées. En revanche, pour chaque domaine spectral, une méthode numérique différente est en général utilisée ; l'unification de ces méthodes représente un défi pour les années à venir afin d'exploiter les divers instruments spatiaux en vol de manière homogène.

De même, pour chaque domaine spectral, des stratégies différentes ont jusque-là été choisies afin de réaliser la montée d'échelle évoquée précédemment. Dans le domaine de l'infrarouge, les derniers codes de transfert radiatif exploitent des ensembles de formes de particules pour la glace nuageuse afin de se rapprocher de la diversité naturelle observée. Les distributions en tailles de particules choisies sont en revanche souvent peu flexibles pour l'utilisateur. À l'inverse, dans le domaine des micro-ondes, les ensembles sont encore peu exploités, mais le choix dans les distributions en tailles de particules est en général rendu très flexible.

Dans ce contexte, la future génération de satellites européens Metop-SG et la $9^{\mathrm{e}}$ mission Earth Explorer devraient permettre de stimuler la communauté scientifique française et internationale sur l'ensemble de ces questions. En effet, les observations conjointes dans les micro-ondes jusqu'aux fréquences submillimétriques (MWI, MWS et ICI), dans les deux domaines infrarouge (instrument Iasi-NG et mission Forum) et dans l'UV-visible polarisé (instrument 3MI) devraient fournir un cadre unique permettant de grandement améliorer la caractérisation des aérosols et des nuages, tout autant que l'étude des surfaces et des espèces gazeuses en atmosphère diffusante.

Hervé Herbin ${ }^{1}$

Philippe Chambon ${ }^{2}$

1. Laboratoire d'optique atmosphérique, Université de Lille / CNRS, Villeneuve d'Ascq 2. Centre national de recherches météorologiques, Université de Toulouse / Météo-France / CNRS / Toulouse

Deguine A., Petitprez D., Clarisse L., Gudmundsson S., Outes V., Villarosa G., Herbin H., 2020. Complex refractive index of volcanic ash aerosol in the infrared, visible, and ultraviolet. Appl. Opt., 59, 884-1252.

Di Biagio C., Boucher H., Caquineau S., Chevaillier S., Cuesta J., Formenti P., 2014. Variability of the infrared complex refractive index of African mineral dust: experimental estimation and implications for radiative transfer and satellite remote sensing. Atmos. Chem. Phys., 14, $11093-11116$.

Eriksson P., Ekelund R., Mendrok J., Brath M., Lemke O., Buehler S.A. 2018. A general database of hydrometeor single scattering properties at microwave and sub-millimetre wavelengths. Earth Syst. Sci. Data, 10, 1301-1326. doi: 10.5194/essd-10-1301-2018

Herbin H., Pujol O., Hubert P., Petitprez D., 2017. New approach for the determination of aerosol refractive indices - Part I: Theoretical bases and numerical methodology. J. Quant. Spectrosc. Radiat. Transfer, 200, 311-319.

Kneifel S., Dias Neto J., Ori D., Moisseev D., Tyynelä J., Adams I.S., Kuo K., Bennartz R., Berne A., Clothiaux E.E., Eriksson P., Geer A.J., Honeyager R., Leinonen J., Westbrook C.D., 2018. Summer snowfall workshop: scattering properties of realistic frozen hydrometeors from simulations and observations, as well as defining a new standard for scattering databases. Bull. Am. Meteorol. Soc., 99, ES55-ES58. doi: 10.1175/BAMS-D-17-0208.1

McPeath R., Bass S., Newnham D., Ballard J., Remedios J., 2002. Comparison of aerosol and thin film spectra of supercooled ternary solution aerosol. J. Geophys. Res., 107, 4371. doi: 10.1029/2001JD000641

Reed B.E., Peters D.M., McPheat R., Grainger R.G., 2018. The complex refractive index of volcanic ash aerosol retrieved from spectral mass extinction. J. Geophys. Res., 123, 1339-1350. 


\section{Application à la météorologie opérationnelle et à la modélisation du climat}

La modélisation de l'atmosphère, élément central des prévisions météorologiques autant que des projections climatiques, consiste à prévoir l'évolution de l'état de l'atmosphère à partir de son état initial. Pour cela, en plus des équations de la mécanique des fluides qui régissent la dynamique à grande échelle, un certain nombre de processus physiques ayant lieu à des échelles inférieures à la maille numérique du modèle doivent être paramétrisés. Parmi eux, les flux radiatifs entre le Soleil, les différentes couches atmosphériques et la surface terrestre sont essentiels, puisqu'ils sont la source primaire d'énergie sur Terre et contrôlent par exemple la température globale moyenne. Très tôt dans la modélisation de l'atmosphère, il a donc fallu développer des outils pour calculer ces flux radiatifs, qui conditionnent le bilan d'énergie de la surface, mais aussi les taux de réchauffement ou de refroidissement atmosphériques via l'absorption et l'émission de rayonnement solaire et infrarouge (annexe 2). Par ailleurs, les modèles météorologiques s'appuient aujourd'hui massivement sur les observations, en particulier satellitaires. Ces observations, via la technique de l'assimilation de données, permettent de corriger l'état initial d'un modèle. Pour cela, des opérateurs simulent les observations à partir des variables du modèle, observations simulées qui sont ensuite comparées aux observations réelles. Dans le cas des observations

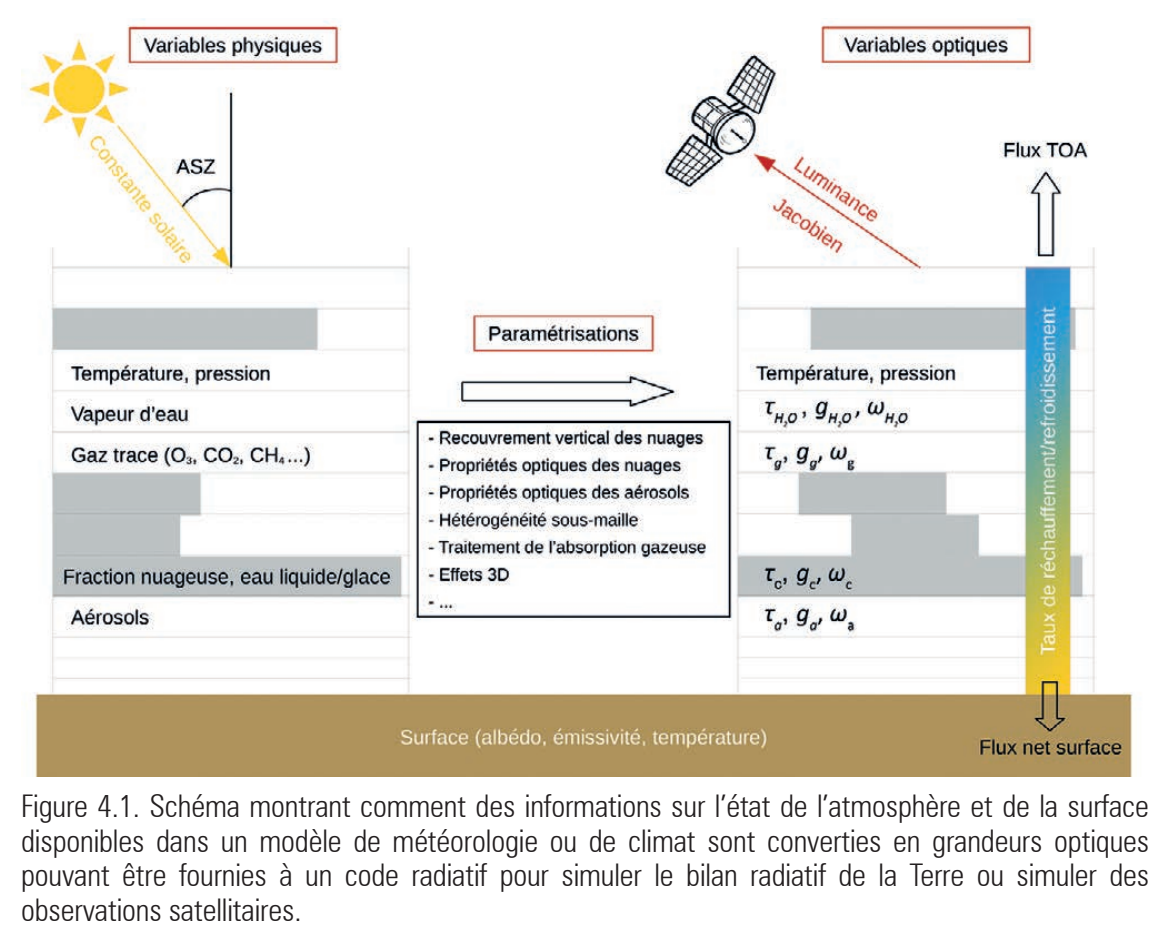

Figure 4.1. Schéma montrant comment des informations sur l'état de l'atmosphère et de la surface disponibles dans un modèle de météorologie ou de climat sont converties en grandeurs optiques pouvant être fournies à un code radiatif pour simuler le bilan radiatif de la Terre ou simuler des observations satellitaires.

satellitaires passives de luminance, qui constituent la grande majorité des observations aujourd'hui assimilées, c'est un code radiatif qui fait cela, celui utilisé à Météo-France - et plus largement en Europe - étant RTTOV (Saunders et al., 2018). Les satellites couvrant tout le spectre électromagnétique, ces codes doivent s'étendre de l'UV aux micro-ondes.

Les codes radiatifs utilisés en météorologie opérationnelle et en physique du climat, pour calculer des flux radiatifs ou des luminances satellitaires, sont généralement sujets aux mêmes enjeux et contraintes. Ces codes doivent avant tout être rapides, puisqu'ils ont vocation à être utilisés en temps réel pour les prévisions à courte échéance et à moyen terme. Le temps de réalisation d'une prévision météorologique devant en effet être plus rapide que le temps réel, cela place d'emblée une contrainte forte sur les performances des codes radiatifs, qui ne sont par ailleurs qu'une composante parmi d'autres de modèles particulièrement complexes. Cette nécessité d'efficacité impose de faire des approximations. Tout d'abord, l'atmosphère est considérée comme une succession de couches parallèles d'extension horizontale infinie, de sorte que chaque colonne du modèle atmosphérique est traitée indépendamment des colonnes voisines. On parle d'approximation des colonnes indépendantes. S'il est manifeste qu'une vision aussi simpliste est rapidement mise en défaut lorsqu'un satellite a une visée oblique qui traverse de nombreuses colonnes adjacentes, ou lorsqu'un nuage voit son ombre déportée sur la colonne voisine, considérer ces échanges horizontaux nécessite une complexification des codes qui va de pair avec un coût de calcul accru, aujourd'hui rédhibitoire. Par ailleurs, les propriétés radiatives de l'atmosphère - en particulier des gaz qui la constituent - varient à très haute fréquence spectrale. Les calculs ne pouvant être réalisés à si haute résolution, différentes approximations sont utilisées. Lorsque les flux intégrés sur de larges bandes spectrales sont la grandeur clé, comme c'est le cas pour les bilans d'énergie, le transfert radiatif est résolu dans un nombre limité de bandes dont on cherche à réduire le nombre sans compromettre la précision des résultats. On utilise pour cela des modèles de bandes ou des $k$-distributions. En pratique, une douzaine de bandes dans le solaire et autant dans l'infrarouge sont utilisées (voir, par exemple, Mlawer at al., 1997). Dans le cas des luminances passives mesurées par satellite, les codes de transfert radiatif utilisent principalement une méthode de paramétrisation linéaire de la transmittance atmosphérique datant du milieu des années 1970, qui permet tout d'abord de gagner en temps de calcul par rapport aux codes raie-parraie de référence, et fournit par ailleurs les jacobiens (dérivées du code direct en fonction des entrées) indispensables aux méthodes variationnelles d'assimilation de données. D'autres approximations permettent de gagner du temps au détriment de la fidélité physique, telle la simplification de la diffusion dans l'infrarouge et les micro-ondes qui s'appuie sur des méthodes à deux flux, la paramétrisation de la fonction de phase des nuages et l'utilisation de climatologies pour certaines espèces gazeuses minoritaires et les aérosols, etc. En dépit de ces approximations, les codes radiatifs restent des composantes gourmandes en ressources au sein des modèles atmosphériques (par exemple, $40 \%$ du temps de calcul de toutes les paramétrisations physiques dans le modèle opérationnel de prévision numérique du temps Arome). Ils visent généralement une précision de l'ordre de $1 \%$ sur des calculs de flux ou à rester en dessous du bruit instrumental des satellites en ciel clair. Cependant, 
des biais permanents, même mineurs, peuvent avoir des conséquences importantes pour les applications climatiques (voir, par exemple, De Angelis, 2015 ; Soden et al., 2018), justifiant le raffinement continu de ces codes.

Une autre spécificité de ces codes radiatifs est qu'ils doivent traiter en entrée des variables physiques souvent éloignées des quantités optiques propres aux codes radiatifs (figure 4.1). L'illustration la plus frappante est le traitement des nuages, composante primordiale du bilan radiatif de l'atmosphère. Dans un modèle atmosphérique, les nuages sont généralement représentés par une fraction nuageuse (proportion d'une maille de modèle qui contient de l'eau condensée) et un contenu en eau condensée (liquide ou glace). Partant de ces informations très partielles, plusieurs étapes sont nécessaires pour calculer l'effet radiatif d'un nuage. Tout d'abord, il faut faire des hypothèses sur le recouvrement vertical des différentes couches nuageuses, qui va déterminer la fraction nuageuse totale vue du sol ou de l'espace. Ensuite, une hypothèse sur la répartition horizontale de l'eau condensée au sein d'une couche doit être faite, partant du constat qu'un nuage n'est jamais homogène à grande échelle et que ses propriétés radiatives sont fortement non linéaires en fonction du contenu en eau. Enfin, les propriétés optiques d'un nuage, en particulier dans le spectre solaire, dépendent largement de la taille des hydrométéores (gouttelettes ou cristaux), qui n'est pas fournie par le modèle atmosphérique. Cela implique de faire une hypothèse sur la distribution de leurs tailles, généralement fondée sur des corrélations entre contenu en eau condensée et rayon effectif observées lors de campagnes de mesure. Dans le cas des nuages de glace, la forme des cristaux doit, elle aussi, être supposée, ce qui constitue une source supplémentaire d'incertitude compte tenu de la complexité et de la diversité des formes observées dans la nature.
Si le transfert radiatif pour les applications météorologiques et climatiques a connu son heure de gloire dans les années 1990, il a été quelque peu mis de côté ces deux dernières décennies, considéré comme un problème résolu $\mathrm{du}$ fait de la connaissance théorique très avancée des processus en jeu, au contraire d'autres processus dont la compréhension physique reste encore limitée, tels que la convection ou la formation des nuages de glace.

Cependant, cette discipline connaît un regain d'intérêt depuis quelques années et l'émergence de nouveaux défis scientifiques. Parmi eux, la nécessité de prendre en compte les échanges radiatifs horizontaux (ou effets 3D, voir annexe 5) en conditions nuageuses. Avec des modèles météorologiques disposant de résolutions horizontales toujours plus fines, ces échanges ne peuvent en effet plus être considérés comme marginaux. Si aujourd'hui les mêmes codes radiatifs sont utilisés à toutes les échelles, des simulations LES (Large-Eddy Simulations, jusqu'à quelques mètres de résolution) aux modèles de climat (plusieurs centaines de kilomètres), des propositions fleurissent pour prendre en compte les effets 3D à coût de calcul raisonnable et il est probable que ces nouveaux modèles seront utilisés d'ici une dizaine d'années en météorologie opérationnelle. On peut citer aussi le défi majeur que représente l'assimilation des luminances satellitaires en conditions nuageuses dans les modèles de prévisions, qui a fait des progrès remarquables dans le domaine des micro-ondes ces dernières années (Geer et al., 2017), mais qui reste encore un défi considérable dans les domaines infrarouge et solaire. Un autre enjeu est l'harmonisation entre les hypothèses faites dans les opérateurs d'observation et les calculs de flux, historiquement développés par des communautés distinctes. Elle va de pair avec une meilleure intégration des codes radiatifs aux autres compartiments de la physique, en premier lieu la microphysique, la turbulence et la convection, qui peuvent fournir des informations utiles sur les structures macro et microscopiques des nuages. Elle nécessite aussi une continuité entre les domaines spectraux qui historiquement ont été abordés indépendamment les uns des autres. Cette nécessité de révision des bases de données des propriétés de diffusion simple des nuages, des aérosols, mais aussi des surfaces (BRDF et émissivités spectrales), accompagne l'avènement de nouvelles missions satellitaires visant à couvrir l'intégralité du spectre électromagnétique, par exemple pour les missions 3MI, ICI, Iasi-NG ou Forum (Libois, 2020). Un dernier enjeu consiste à l'ajout de variables pronostiques dans les modèles météorologiques, par exemple la concentration en hydrométéores, le contenu en aérosols de diverses espèces ou la concentration en ozone, afin de mieux représenter les interactions et rétroactions entre l'ensemble des agents radiatifs de l'atmosphère.

Longtemps considérés comme des boîtes noires représentant des processus complexes mais bien compris, et par conséquent abandonnées aux seuls experts, les codes radiatifs reviennent aujourd'hui sur le devant de la scène. C'est de la complémentarité de nouvelles mesures (en laboratoire, in situ et satellitaires), de développements de paramétrisations originales et des échanges accrus avec les autres développeurs des modèles atmosphériques que des progrès significatifs seront réalisés dans la compréhension du rôle des transferts radiatifs en météorologie et pour le climat.

Quentin Libois
Jérôme Vidot
Centre national de recherches météorologiques,
Université de Toulouse / Météo-France/
CNRS / Toulouse

DeAngelis A.M., Qu X., Zelinka M.D., Hall A., 2015. An observational radiative constraint on hydrologic cycle intensification. Nature, 528, 249-253. Geer A.J., Lonitz K., Weston P. et al., 2018. All-sky satellite data assimilation at operational weather forecasting centres. Q. J. R. Meteorol. Soc., 144, 1191-1217. doi: 10.1002/qj.3202

Libois Q., C.-Labonnote L., Camy-Peyret C., 2020. Forum mesurera l'infrarouge lointain émis par la Terre. La Météorologie, 108, 4-6.

Mlawer E.J., Taubman S.J., Brown P.D., Iacono M.J., Clough S.A., 1997. Radiative transfer for inhomogeneous atmospheres: RRTM, a validated correlated-k model for the longwave. J. Geophys. Res. Atmos., 102, 16663-16682.

Saunders R., Hocking J., Turner E., Rayer P., Rundle D., Brunel P., Vidot J., Roquet P., Matricardi M., Geer A., Bormann N., Lupu C., 2018. An update on the RTTOV fast radiative transfer model (currently at version 12). Geosci. Model Dev., 11, 2717-2737. doi: 10.5194/gmd-11-2717- 2018 


\section{Modélisation tridimensionnelle du transfert radiatif pour des scènes terrestres naturelles}

Reproduire visuellement et/ou quantitativement les scènes géophysiques naturelles est un défi, du fait des multiples phénomènes physiques (absorption, diffusion, émission, polarisation...) à prendre en compte à toutes les échelles spatio-temporelles. Le rayonnement solaire ou thermique interagit avec l'ensemble des composants atmosphériques (gaz, aérosols, gouttelettes d'eau, cristaux de glace...) et de surface (océan, désert, canopée, bâtiments urbains...) dont la complexité est d'autant plus grande que l'échelle spatiale est fine. Des modèles physiques (fondés sur l'équation intégro-différentielle du transfert radiatif unidimensionnel (TR1D) où les paramètres géophysiques ne varient que selon la verticale) et mathématiques (approximation à deux flux, aux ordonnées discrètes ...) ont été mis en place depuis les années 1950 afin d'estimer le rayonnement. Cependant, ces paramètres varient dans les trois dimensions de l'espace (concentration en eau des nuages, par exemple) et le calcul des propriétés radiatives (flux, taux de chauffage, luminance...) nécessite des codes de transfert radiatif tridimensionnel (TR3D). Le but de cet article est de dresser une présentation générale des techniques de modélisation du TR3D tout en soulignant les difficultés et les limites de ces approches en fonction des domaines d'application.

\section{Les approches de modélisation du rayonnement atmosphérique tridimensionnel}

Les codes de TR3D sont très coûteux en ressources informatiques, puisqu'ils doivent résoudre l'ETR (équation du transfert radiatif) pour toutes les directions de l'espace dans un milieu 3D. Il existe deux approches : les méthodes de Monte Carlo (simulation d'un grand nombre de «trajectoires de photons ») issues de la neutronique et adaptées dans les années 1980 à l'atmosphère, et les méthodes déterministes (le champ de rayonnement est explicitement représenté de manière discrète). Ces deux approches de référence sont généralement utilisées pour valider des approches simplifiées, moins précises, mais plus rapides.
L'approximation du TR3D la plus usitée est l'approximation du pixel ou de la colonne indépendante (IPA) : pour chaque pixel d'une image satellitaire ou pour chaque colonne d'un modèle atmosphérique, les propriétés radiatives sont calculées à l'aide d'un code de TR1D. Certains processus physiques ne sont pas décrits, comme les interactions radiatives horizontales (c'est-à-dire « le transport horizontal des photons ») entre le milieu nuageux et l'atmosphère claire, par exemple. Ceci peut conduire à des scènes modélisées présentant de forts gradients de rayonnement irréalistes sur les bords de nuages.

La figure 5.1 décrit l'ensemble des phénomènes radiatifs à prendre en compte pour une scène nuageuse tridimensionnelle.

\section{Les applications en sciences atmosphériques}

Les codes de TR3D approximés qui reposent sur des améliorations successives de l'IPA depuis les années 1990 trouvent de nombreuses applications dans le problème direct (simulation des flux, des taux de chauffage dans les modèles de climat, de prévision numérique du temps, dans les modèles de simulation aux grandes échelle ou LES (Large Eddy Simulation)...) et dans le problème inverse (restitution des produits atmosphériques pour la surveillance de l'état des cultures, pour la détermination de la composition des couverts végétaux ou des milieux nuageux, par télédétection passive ou active au sol, aéroportée ou satellitaire...). Ces modèles simplifiés sont souvent mis en œuvre dans les algorithmes opérationnels, afin d'accroître la rapidité des calculs, en associant une précision des résultats compatible avec les besoins. Ces modèles sont aussi utilisés dans d'autres applications comme la médecine, l'étude des échanges thermiques dans les moteurs ou les bâtiments.

Les codes de TR3D de référence reposent généralement sur une approche explicite, comme SHDOM (Evans, 1998) ou statistique de type Monte Carlo, comme 3DMCPOL (Cornet et al., 2010). Ils sont plutôt utilisés dans les laboratoires de recherches pour réaliser des études de sensibilité du rayonnement aux propriétés des constituants atmosphériques afin de comprendre les effets radiatifs $3 \mathrm{D}$, d'évaluer leurs

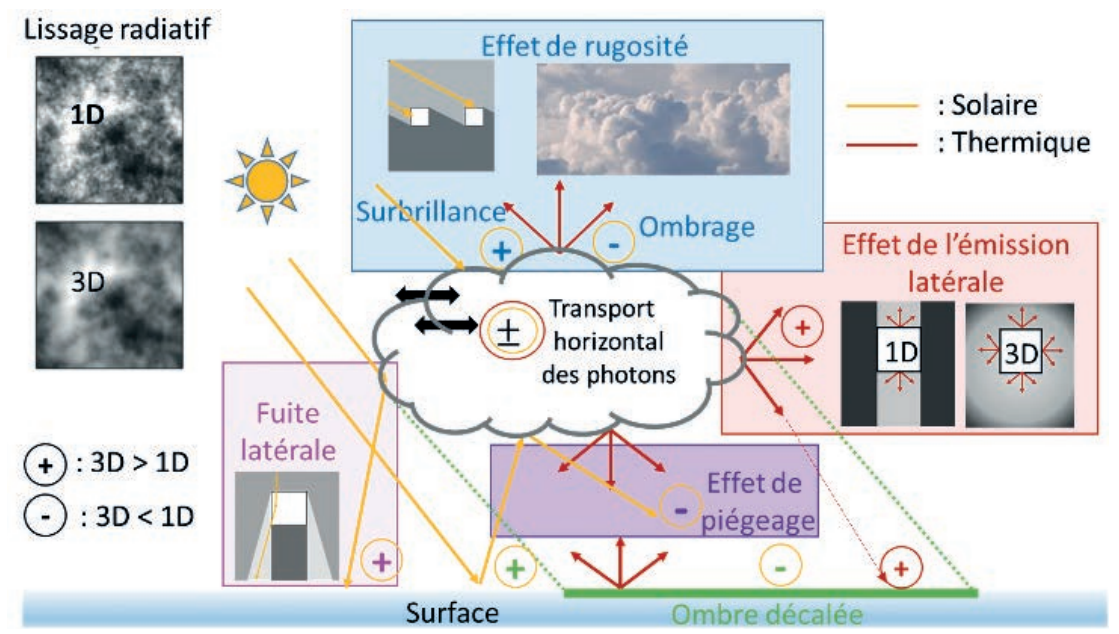

Figure 5.1. Illustration des principaux effets radiatifs 3D des nuages. La théorie du transfert radiatif 1D, par essence, ne prend pas en compte le transport horizontal des photons (flèches noires horizontales), dont les effets sont plus importants dans le domaine visible (traits jaunes) que thermique (traits rouges). Dans le visible, ce processus est à l'origine du lissage radiatif (quand le soleil est au zénith, le champ de luminances 3D d'un nuage est plus lisse qu'en 1D), de l'effet de rugosité (quand l'angle d'incidence solaire est élevé, le champ de radiance 3D apparaît plus rugueux qu'en $1 \mathrm{D}$ en lien avec les phénomènes de surbrillance et d'ombrage), de l'effet de fuite en bord de nuage (contribuant par exemple à une augmentation de l'éclairement au sol) et à l'effet de piégeage (des photons peuvent être piégés entre la base du nuage et le sol ou entre différentes couches nuageuses et ne plus contribuer à la luminance zénithale). Dans le thermique, le principal effet radiatif 3D des nuages est l'émission thermique du bord des nuages qui peut contribuer, par exemple, à une augmentation significative du rayonnement à la surface. 
impacts et de proposer des corrections des algorithmes opérationnels. Ils sont aussi nécessaires à la mise au point des traitements de détection de cibles dans le domaine de la défense et pour le rendu de scènes réalistes visuellement et/ ou physiquement.

\section{Difficulté du TR3D}

Une des limites des codes de TR3D de référence est le besoin de puissances de calcul colossales (temps et charge mémoire). Des méthodes de réduction de variance ont été développées pour les méthodes Monte Carlo, mais elles ne sont pas toujours efficaces dans certaines configurations (lidar, par exemple). L'autre limite des codes de TR3D est la méconnaissance des propriétés spatiales des scènes géophysiques terrestres. Par exemple, on ne connaît pas comment fluctuent, en fonction de l'échelle spatiale, toutes les propriétés microphysiques des nuages. En effet, les mesures in situ ou de télédétection n'apportent que des informations parcellaires à différentes échelles spatio-temporelles. Bien sûr, la communauté scientifique met au point différents modèles mathématiques et/ou physiques afin de renseigner les propriétés de certains éléments composant la scène (par exemple modèle fractal ou simulation LES pour la génération de nuages). Mais la méconnaissance de certains phénomènes physiques entraîne une incertitude dans les entrées des modèles de TR3D qui influent sur la précision des grandeurs radiatives calculées et également sur le rendu de ces scènes.

\section{Quel avenir pour les scènes radiatives tridimensionnelles}

Des études récentes ont montré que les effets radiatifs tridimensionnels ont un impact sur l'évolution de la structure des nuages de type stratocumulus. Ceci révèle l'importance de quantifier l'impact du TR3D sur la microphysique et le cycle de vie des nuages et nécessitera l'implémentation de codes de TR3D précis mais rapides dans les LES.

De nombreuses études ont montré que les hétérogénéités verticales et horizontales des nuages à l'échelle des colonnes des modèles climatiques globaux ont un impact sur les taux de réchauffement. Il est donc légitime de savoir si ces hétérogénéités peuvent avoir un impact sur les projections climatiques de ces modèles. Des efforts sont actuellement menés au sein de la communauté internationale afin d'adapter des codes TR3D pour ces modèles.

De nombreuses études ont montré que les produits nuageux inversés pixel par pixel par capteurs passifs embarqués sur plateforme satellite étaient biaisés par les effets 3D des nuages. Il est primordial de développer de nouveaux concepts (tomographie par exemple) pour inverser directement des scènes nuageuses. Ce type d'approche nécessitera certainement la fusion de données issues de capteurs de différentes natures (actif, passif, résolutions spatiales). Par exemple, un des objectifs de la future mission spatiale franco-israélienne $\mathrm{C}^{3}$ IEL (Cluster for Climate and Cloud Imaging of Evolution and Lightning) est de restituer l'enveloppe nuageuse à très haute résolution spatiale par triangulation de trois caméras embarquées sur trois nanosatellites. Ces algorithmes de restitution seront obligatoirement fondés sur des codes de TR3D.

Les progrès des calculateurs permettront d'accroître la rapidité des calculs tout en mettant en œuvre la capacité de décrire les scènes naturelles avec de très nombreux paramètres d'une manière tridimensionnelle. Notre connaissance du milieu naturel atmosphérique à différentes échelles spatiales et temporelles est incomplète et des efforts seront à mener sur l'utilisation de modèles physiques et/ ou mathématiques permettant de renseigner les grandeurs en entrée des codes radiatifs pour pallier ce manque de connaissances précises, ainsi que d'analyses de campagnes expérimentales. Les besoins civils ou militaires sont de plus en plus importants pour ces scènes 3D et l'avenir verra une généralisation de modèles $3 \mathrm{D}$ rapides dont la précision aura été estimée par les modèles de référence, mais qui seront suffisants pour les besoins visés.

Patrick Chervet $^{1}$
Frédéric Szczap $^{2}$
1. Onera, Palaiseau
2. Laboratoire de météorologie physique,
Université Clermont Auvergne, Aubière

Cornet C., C-Labonnote L., Szczap F., 2010. Three-dimensional polarized Monte Carlo atmospheric radiative transfer model (3DMCPOL): 3D effects on polarized visible reflectances of a cirrus cloud. J. Quant. Spect. Radiat. Transfer, 111, 174-186. doi: 10.1016/j.jqsrt.2009.06.013

Evans K.F., 1998. The spherical harmonic discrete ordinate method for three-dimensional atmospheric radiative transfer. J. Atmos. Sci., 55, $429-446$. 


\section{Utilisation du transfert radiatif par les agences spatiales}

Les agences spatiales, comme le Cnes ou Eumetsat, sont en charge de la conception, du développement et de l'exploitation de missions spatiales. Comme l'illustre la figure 6.1, elles font ainsi le lien entre le besoin des utilisateurs (souvent exprimé via une question scientifique) et le maître d'œuvre industriel qui réalise le satellite et le système ${ }^{1}$. Pour cela, les agences conduisent dans leurs centres techniques des activités liées à la physique de la mesure de télédétection, que ce soit pour la prise en compte du besoin des utilisateurs (besoins liés à la recherche scientifique, aux applications civiles ou aux systèmes opérationnels comme la prévision du temps), la définition et l'amélioration des algorithmes de traitement, l'étalonnage des capteurs ou la validation des produits.

Les missions spatiales concernées sont à vocation environnementale ou scientifique et couvrent l'imagerie spatiale, le sondage atmosphérique passif (spectroscopie) et actif (lidar). Nous traiterons ici en particulier des domaines spectraux visibles et infrarouge, même si les agences sont également impliquées dans des activités de physique de la mesure dans le domaine micro-ondes, passif ou actif.

\section{L'utilisation du transfert radiatif dans les projets spatiaux}

Le transfert radiatif est un outil phare pour toutes les étapes des projets spatiaux, depuis les études de concept et de faisabilité jusqu'à l'exploitation en vol, et fait également l'objet d'études transverses en support aux projets. En particulier, il est fait appel à des codes de transfert radiatif pour :

- la modélisation du signal en entrée de l'instrument : quantification du niveau de flux ou degré de polarisation par exemple pour le design du capteur, analyse du contenu en information géophysique pour vérifier/ajuster un design ou proposer de nouveaux concepts...;

- l'estimation de la performance des mesures et des produits : calcul de la précision de mesure pour le dimensionnement d'un instrument, en prenant en compte l'impact des paramètres géophysiques (composition de l'atmosphère, type de surface, etc.) ;

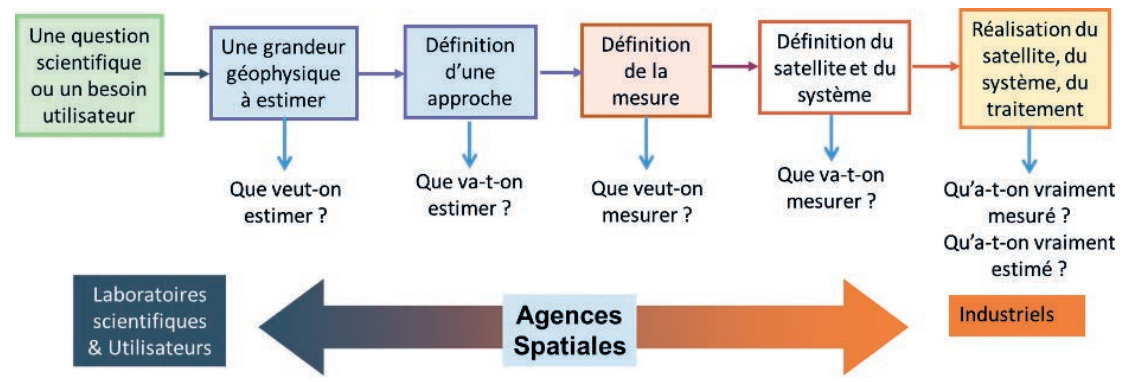

Figure 6.1. Rôle des agences spatiales.

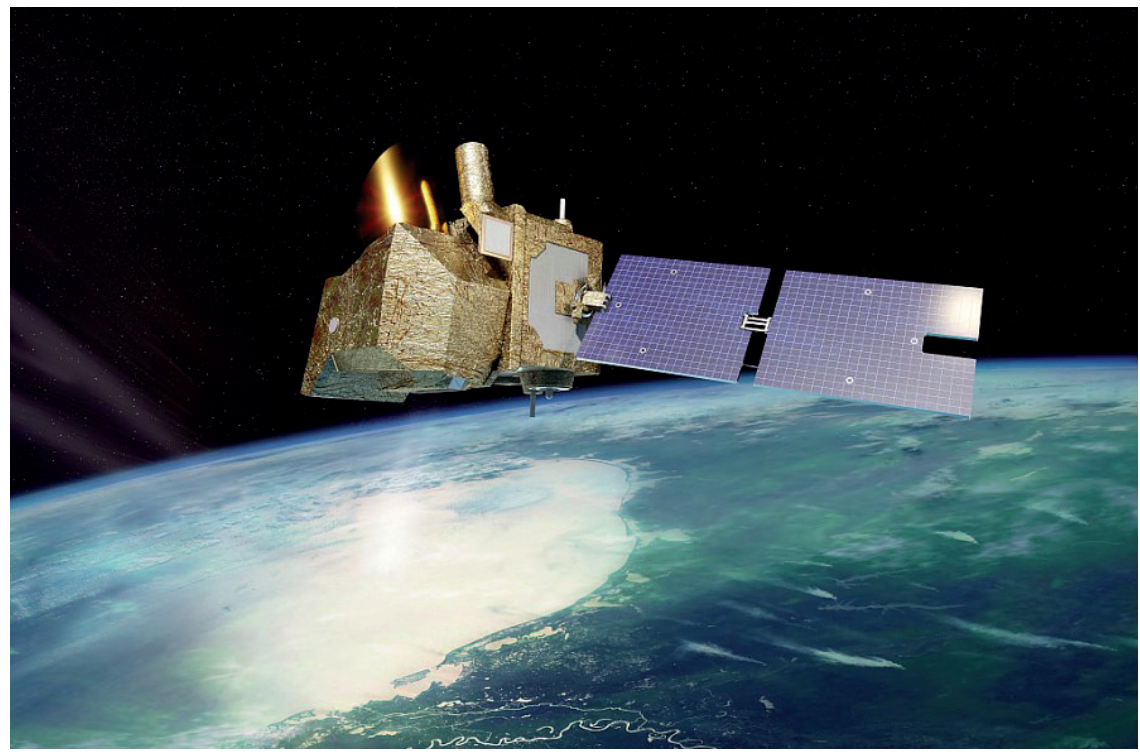

Figure 6.2. Vue d'artiste de MicroCarb.

- la définition des algorithmes de traitement des données : par exemple l'inversion des paramètres géophysiques pour la préparation et le développement des segments sol de la mission;

- l'exploitation des mesures et des produits une fois la mission en vol : pour l'étalonnage des capteurs sur satellite, l'exploitation des mesures du satellite pour les traduire en paramètres géophysiques, l'exploitation d'instruments terrain ou de mesures aéroportés pour la validation, le suivi en vol de la mission, etc.

La diversité des usages est combinée à une grande variété de thématiques et d'observables à la fois pour l'atmosphère (météorologie, gaz à effet de serre, chimie atmosphérique, aérosols, nuages...) et les surfaces (biosphère, " couleur de l'eau », terres émergées, température de surface, glace de mer). Par ailleurs, les besoins en termes d'outils de transfert radiatif sont sans cesse renouvelés avec de nouvelles missions à l'étude chaque année. In fine, tout cela explique le besoin des agences spatiales de disposer de nombreux codes « prêt à l'emploi », avec toujours plus de fonctionnalités, à la fois raie-par-raie ou par modèle de bandes, rapides ou très précis, prenant en compte la diffusion et/ou l'absorption, la polarisation, des modèles de surface élaborés, le calcul des jacobiens, etc.

\section{Travaux récents et perspectives}

\section{Au Cnes}

Parce qu'il va être utilisé opérationnellement dans les segments sols des futures missions du Cnes que sont MicroCarb (figure 6.2) et Merlin, le code de référence $4 \mathrm{~A} / \mathrm{OP}$ a été mis à niveau en incluant de nouvelles fonctionnalités. Par ailleurs, afin d'en faire bénéficier la communauté scientifique, 4A/OP est depuis avril 2017 un logiciel

1. Ensemble des éléments qui constituent la mission spatiale (instrument de mesure - ou charge utile - et plate-forme, mais aussi orbite, segment sol, traitements, opérations, etc.). 
libre sous licence LGPL, distribué par le pôle de données Aeris² ${ }^{2}$. De même, les codes SOS et OSOAA développés par le Cnes sont passés sous licence GPL $^{3}$ et distribués librement ${ }^{4}$. Enfin, le Cnes a récemment élargi sa bibliothèque d'outils de transfert radiatif pour les missions actives, avec le développement de BLISS (Backscattering Lidar Signal Simulator).

Sont prévus des travaux sur l'accélération des codes, le transfert radiatif simplifié, de nouvelles fonctionnalités pour les projets (par exemple la fluorescence $^{5}$ ), l'extension vers le domaine visible et ultraviolet pour 4A/OP ; avec toujours une prise en compte des besoins de la communauté scientifique : licences libres, distribution facilitée, implémentation de fonctionnalités demandées par les laboratoires, etc.

\section{À Eumetsat}

RTTOV est un code particulièrement important pour les applications opérationnelles atmosphériques. Il a la particularité d'être utilisé par l'ensemble des acteurs, depuis l'agence spatiale jusqu'aux centres d'exploitation et les centres météorologiques, un de ces principaux atouts étant la rapidité de calcul pour les besoins des applications temps réel. Ce code, développé par une communauté d'utilisateurs, est toujours en évolution et permet une importante rationalisation des efforts.

\section{2. https://4aop.aeris-data.fr/}

3. Acronyme de General Public Licence, type de licence qui fixe les conditions de distribution d'un logiciel libre.

4. https://github.com/CNES/

5. Il s'agit ici de la lumière émise par la végétation lorsqu'elle est soumise au rayonnement solaire.

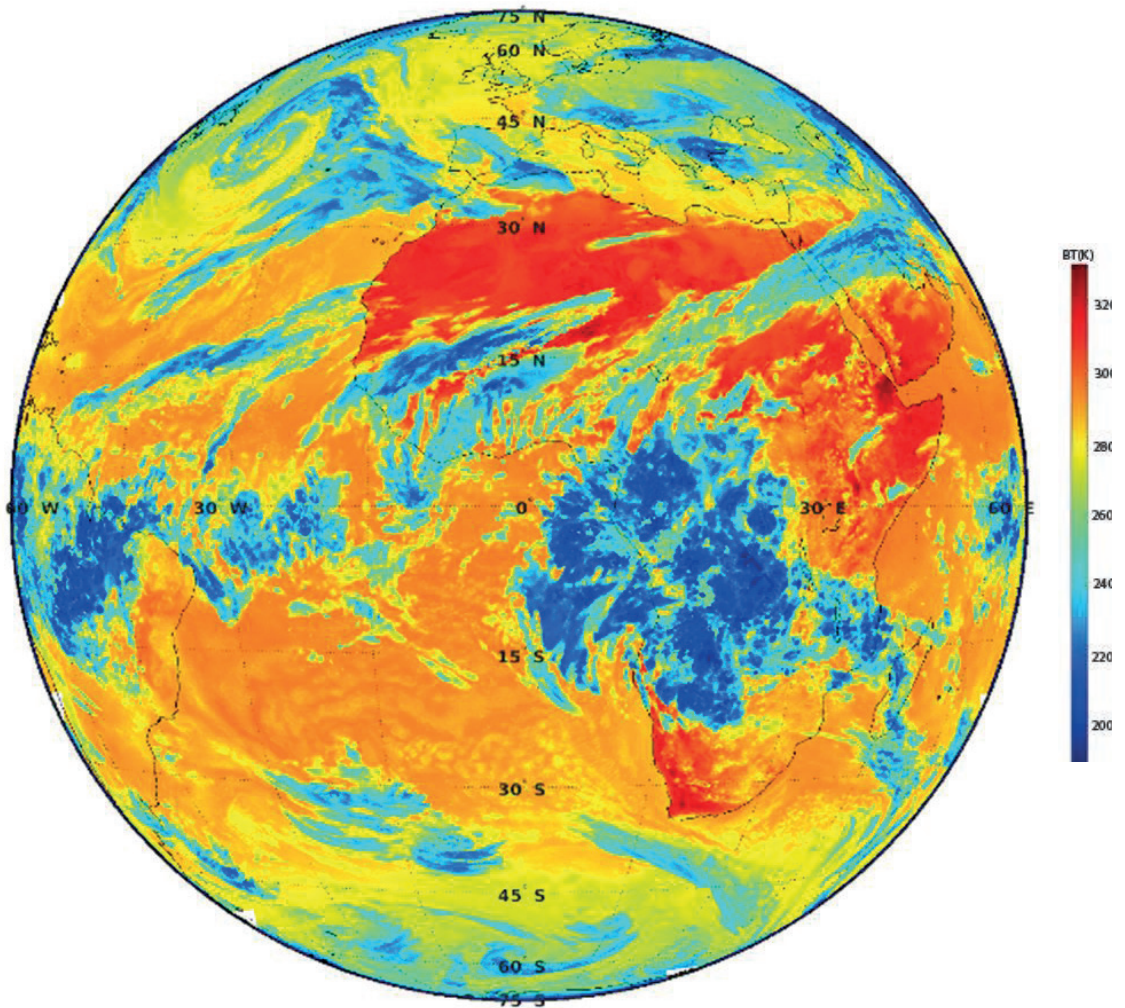

Figure 6.3. Simulation très réaliste des tempé- ratures de brillance BT (ici at $900 \mathrm{~cm}^{-1}$ ) qu'observerait IRS/MTG pendant un cycle de 24 heures. L'échelle de couleur est présentée en kelvins.

Les systèmes spatiaux en développement, tels que $M T G, E P S-S G$ ou Sentinel, ont aujourd'hui besoin de s'appuyer sur des simulations très réalistes des données qui seront acquises de manière à développer et préparer l'exploitation de ces missions (figure 6.3). Sont par exemple utilisés : ArtDeco/Disort pour les capteurs VII (Visible Infrared Imager from EPSSG), 3MI et FCI (Flexible Combined Imager), RTTOV-IRS pour IRS (Infrared Sounder), RTTOV pour FCI et VII, SOS et Sciatran pour 3MI, etc.
Les développements à venir seront dictés par les besoins futurs, notamment : accélération des codes, prise en compte de la polarisation, amélioration des bases spectroscopiques, de la performance en général, harmonisation des codes utilisés à travers les missions, etc.

\section{Clémence Pierangelo ${ }^{1}$} Bertrand Fougnie ${ }^{2}$

1. Cnes, Centre spatial de Toulouse, Toulouse 2. Eumetsat, Darmstadt, Allemagne 